\title{
Die körperliche Untersuchung im Rahmen des Kinder- und Jugendgesundheitssurveys
}

\author{
A. Dippelhofer \\ K. E. Bergmann \\ H. Kahl \\ M. Lange
}

\author{
Physical Examination in the National Health Interview and Examination Survey \\ for Children and Adolescents
}

\section{Zusammenfassung}

Der Kinder- und Jugendgesundheitssurvey hat die umfassende Beschreibung der gesundheitlischen Situation von Kindern und Jugendlichen in Deutschland zum Ziel. Die medizinisch-physikalische Untersuchung der Kinder und Jugendlichen ermöglicht dabei die Erhebung objektiver Befunde zum körperlichen Entwicklungsstand, zu bestimmten Körperfunktionen und ausgewählten Leistungsparametern. Im Vordergrund stehen Indikatoren, die auf zunehmende Entwicklungs- und Gesundheitsprobleme im Kindes- und Jugendalter hinweisen und zum Teil einen prädiktiven Aussagewert für den Gesundheitszustand im Erwachsenenalter haben. Es werden Körpermesswerte einschließlich des Reifestatus erhoben, der Blutdruck gemessen, Sehtests durchgeführt und die koordinativen Fähigkeiten sowie die Ausdauerleistung überprüft. Darüber hinaus wird bei akuten Veränderungen der Schweregrad der atopischen Dermatitis erfasst. Die Methoden und Erhebungsinstrumente wurden in einem einjährigen Pretest überprüft und für die Hauptphase der Studie optimiert.

\section{Schlüsselwörter}

Blutdruckmessung · Sehtest · Anthropometrie $\cdot$ motorische Tests . Kinder und Jugendliche

\section{Abstract}

The National Health Survey for Children and Adolescents intends a comprehensive description of the health status of children and adolescents. In this context physical examination of children and adolescents permits collection of objective data concerning physical development, certain body functions and selected performance parameters. Most important are indicators pointing towards increasing developmental and health problems in children and adolescents, which are partly predictive for the health status as an adult. Body measurements including stages of puberty will be taken, the blood pressure measured, vision tests carried out, coordination skills and endurance tested. Besides, if acute symptoms are detected, severity of atopic dermatitis is recorded. The methods and instruments have been tested in a pilot study over a period of one year and were optimised for the main study.

\section{Key words}

Blood pressure measurement · vision test · anthropometry · motor tests $\cdot$ children and adolescents

\section{Einleitung}

Der Kinder- und Jugendgesundheitssurvey hat das Ziel, verallgemeinerungsfähige, umsetzungsrelevante Daten und Erkenntnisse zur gesundheitlichen Situation von Kindern und Jugendlichen im Alter zwischen 0 und 18 Jahren in Deutschland zu gewinnen. Dabei werden in einem Zeitraum von drei Jahren ca. 18000 Kinder und Jugendliche untersucht. Zusätzlich zur schriftlichen und mündlichen (ärztlichen) Befragung werden Befunde erhoben, die von der subjektiven Einschätzung der untersuchten Probanden und ihrer Eltern unabhängig sind. Die körperlichen Untersuchungen konzentrieren sich dabei auf 
Bereiche, in denen wesentliche Gesundheitsprobleme auftreten. Die Schwerpunkte der Untersuchung und der Einsatz der Methoden werden von den entwicklungsspezifischen Besonderheiten in den jeweiligen Altersgruppen bestimmt. Die ausgewählten Erhebungsinstrumente sind in einem Pretest von März 2001 bis März 2002 an 1629 Kindern und Jugendlichen unter inhaltlichen und methodischen Gesichtspunkten überprüft und für die Hauptphase des Kinder- und Jugendgesundheitssurveys modifiziert worden. Die in Tab. 1 detailliert aufgeführten Untersuchungen und Tests haben sich in der Pilotphase als Screening-Instrumente bewährt und tragen darüber hinaus auch den räumlichen und zeitlichen Beschränkungen des Studiendesign Rechnung.

Tab. 1 Übersicht über die körperlichen Untersuchungen

\begin{tabular}{|c|c|c|}
\hline Sehtests & Blutdruck/Puls & Motoriktests \\
\hline $\begin{array}{l}\text { 3-17 Jahre } \\
\text { - Lang-Test } \\
\text { 3-6 Jahre } \\
\text { - H-Test } \\
\text { 7-17 Jahre } \\
\text { - C-Test mit Einzel- und } \\
\text { Reihenoptotypen }\end{array}$ & $\begin{array}{l}\text { 3-17 Jahre } \\
\text { - mittlerer arterieller } \\
\text { systolischer und } \\
\text { diastolischer Blutdruck } \\
\text { in Ruhe } \\
\text { - Ruhepuls }\end{array}$ & $\begin{array}{l}\text { 4-10 Jahre } \\
\text { - Einbeinstand } \\
\text { - seitliches Hin- und } \\
\text { Herspringen } \\
\text { - Liniennachfahren } \\
\text { - Stifte umstecken } \\
\text { - Reaktionstest } \\
\text { - Rumpfvorbeuge } \\
\text { 11-17 Jahre } \\
\text { - Fahrradergometrie }\end{array}$ \\
\hline Hautuntersuchung & Anthropometrie & Reifestatus \\
\hline $\begin{array}{l}\text { 0-17 Jahre } \\
\text { - Ausdehnung und } \\
\text { Schweregrad der } \\
\text { akuten atopischen } \\
\text { Dermatitis nach } \\
\text { Scorad }\end{array}$ & $\begin{array}{l}\text { 0-17 Jahre } \\
\text { - Körperlänge } \\
\text { - Körpergewicht } \\
\text { - Kopfumfang } \\
\text { - Hautfaltendicke } \\
\text { 6-17 Jahre } \\
\text { - Epikondylenbreite } \\
\text { 11-17 Jahre } \\
\text { - Taillenumfang } \\
\text { - Hüftumfang }\end{array}$ & $\begin{array}{l}\text { 10-17 Jahre } \\
\text { - Entwicklungsstufen de } \\
\text { Pubesbehaarung } \\
\text { - Regelblutung } \\
\text { - Stimmbruch }\end{array}$ \\
\hline
\end{tabular}

\section{Sehtest}

\section{Problemstellung}

Die Häufigkeit von Sehminderungen durch Refraktionsfehler im Kindesalter wird auf ca. 15-30\% [Moore 1998] geschätzt. Die Datenlage auf Bevölkerungsebene ist in Deutschland nicht ausreichend, da u.a. die Sehschärfebestimmungen im Rahmen der Vorsorgeuntersuchungen der Krankenkassen und der Schuluntersuchungen durch die Gesundheitsämter nicht methodisch einheitlich erfolgen (unterschiedliche Sehtafeln bzw. Sehtestgeräte) und sich auf bestimmte Altersgruppen beschränken [Hohmann, Haase 1993]. Neben den Refraktionsfehlern, die durch eine Brille korrigierbar sind, spielt im Kindesalter die Entdeckung von Amblyopien, d. h. die Schwachsichtigkeit meist eines Auges, eine große Rolle, da dieser Sehfehler nur bis zum vollendeten vierten Lebensjahr gut therapierbar ist. Die geschätzte Prävalenz dieser zumindest prinzipiell vermeidbaren Erkrankung beträgt im Kindesalter 2-4\% [Moore 1998].

Bei den Sehtests, die im Rahmen des Kinder- und Jugendgesundheitssurveys durchgeführt werden, steht die differenzierte Beschreibung der Verbreitung von Sehstörungen einschließlich des Ausgleichs durch Sehhilfen im Vordergrund. Die Daten sollen eine Grundlage für regionale, internationale und zeitliche Ver- gleiche (Trends) bilden. Außerdem soll ermittelt werden, welche Beziehungen zwischen Sehstörungen und Schulleistungen, motorischen Fähigkeiten, Unfallverletzungen, Lebensqualität, Inanspruchnahme medizinischer Leistungen und - davon abgeleitet Gesundheitskosten bestehen.

\section{Methode und Stichprobe}

Das räumliche Sehen wird bei allen Kindern ab drei Jahren mit dem Lang-Test geprüft. Bei diesem Stereo-Test sollen die Kinder aus $40 \mathrm{~cm}$ Entfernung auf einer Tafel (hergestellt im Zylinderrasterverfahren) Gegenstände benennen, die bei Fehlen des räumlichen Sehens nicht erkennbar sind. Die Testung des Fernvisus erfolgt bei den 3- bis 6-Jährigen mit dem H-Test, der altersangepasst mit vier Sehzeichen auf der Grundlage eines musikalischen Feedbacks arbeitet. Bei den über 6-Jährigen werden Fern- und Nahvisus mit dem C-Test (Landolt-Ringe, Einzel- und Reihenoptotypen) bestimmt.

\section{Erfahrungen aus dem Pretest}

Im Pretest konnte die grundsätzliche Eignung der ausgewählten Methoden und Erhebungsinstrumente bewiesen werden; die gewonnenen Erfahrungen führten zu einer Verbesserung und Präzisierung des Instrumentariums hinsichtlich der Ausleuchtung der Sehtafeln und der Fixierung des Kopfes bei der Testung des Nahvisus.

\section{Blutdruck- und Pulsmessung}

\section{Problemstellung}

Erhöhter Blutdruck ist ein Risikofaktor für Herz-Kreislauf-Erkrankungen und zerebrale Schlaganfälle. Studien aus den USA haben auch bei Kindern und Jugendlichen einen signifikanten Zusammenhang zwischen systolischem und diastolischem Blutdruck und den Serumkonzentrationen von Lipiden, Lipoproteinen und Apolipoproteinen nachgewiesen [Stallones et al. 1982]. Es gibt mehrere Studien, die zeigen, dass die Entwicklung der essenziellen Hypertonie in der Kindheit beginnt und eine Korrelation zwischen dem Blutdruck in der frühen Kindheit, der Adoleszenz und dem Erwachsenenalter besteht [De Swiet 1992, Lauer, Clarke 1989, Nelson et al. 1992]. In Deutschland gibt es zur Problematik des kardiovaskulären Risikos bei Kindern und Jugendlichen besonders unter dem Blickwinkel der zunehmenden Adipositas wenige Untersuchungen [Sönnichsen et al. 1997]. Bundesweit repräsentative Daten zur Prävalenz von hohem Blutdruck bei Kindern und Jugendlichen und zum Zusammenhängen mit anderen Risiken und/oder soziodemografischen Merkmalen fehlen in der Literatur.

Mit der Messung des Blutdrucks im Rahmen des Kinder- und Jugendgesundheitssurveys kann die Verbreitung eines der wichtigsten Risikofaktoren für spätere Herz-Kreislauf-Krankheiten nach Alter, Geschlecht, Region, Gemeindegrößenklasse, Sozialstatus und anderen differenzierenden Variablen beschrieben werden. Zusammenhänge mit den Körpermaßen, den motorischen Fähigkeiten, serologischen Risikofaktoren, Indikatoren des Lebensstils und Präventionsverhaltens sowie anderen relevanten Variablen lassen sich analysieren. Durch die Standardisierung der Methodik sollen die Daten auch eine verlässliche Grundlage für spätere Trendbeobachtungen abgeben. 


\section{Methode und Stichprobe}

Die Blutdruckmessung wird bei allen Studienteilnehmern ab drei Jahren vorgenommen. Die Untersuchung erfolgt unter standardisierten Bedingungen zweimal im Abstand von zwei Minuten mit einem automatischen Gerät (Oszillometer). Dabei werden der Puls und der arterielle Mitteldruck gemessen und der systolische und diastolische Blutdruck nach gerätespezifischen Algorithmen errechnet.

\section{Erfahrungen aus dem Pretest}

Zur Optimierung des Vorgehens bei der Blutdruckmessung wurden in der Pilotphase des Kinder- und Jugendgesundheitssurveys die auskultatorische Methode nach Riva Rocci und die Oszillometrie unter Berücksichtigung der Vorgaben durch das Studiendesign und die Altersstruktur der Teilnehmer hinsichtlich ihrer Validität und Plausibilität verglichen. Die Quecksilbermanometermethode hat sich dabei aufgrund der für eine exakte Auskultation ungünstigen Feldbedingungen (Umgebungslärm, Unruhe durch das Kind oder Begleitpersonen) sowie der für die Hauptphase der Studie erforderlichen Standardisierung von mehreren Untersuchern mit unterschiedlichen persönlichen Voraussetzungen als ungeeignet erwiesen. Die automatische Blutdruckmessung mit einem Oszillometer bietet hingegen die Möglichkeit, über das gesamte kindliche Altersspektrum zuverlässig den Blutdruck zu messen, und ist die derzeit in der klinischen Praxis am weitesten verbreitete Methode.

\section{Motorische Tests}

\section{Problemstellung}

Die Einschränkung kindlicher Bewegungserfahrungen durch gesellschaftliche Veränderungen (Technisierung der Umwelt, Medienkonsum etc.) zeigt sich in Veränderungen des Bewegungsverhaltens und der motorischen Entwicklung der Kinder und Jugendlichen und hat damit Einfluss auf das gesamte kindliche Entwicklungsgeschehen [Gaschler 1999, Etnier et al. 1997]. Folgen des Bewegungsmangels dokumentieren sich heute in einer Zunahme von motorischen und anderen Entwicklungsauffälligkeiten, dem Anstieg des Anteils übergewichtiger Kinder und Kindern mit Diabetes mellitus und weisen auf das Auftreten so genannter Zivilisationskrankheiten bereits bei Kindern hin [Mensink et al. 1996, Zieske et al. 2002]. Aus schulärztlichen und sportmedizinischen Untersuchungen liegen Daten vor, die auf eine Zunahme von motorischen Auffälligkeiten, von Muskelschwächen und einer eingeschränkten Ausdauerleistungsfähigkeit hinweisen. Die Ergebnisse sind aufgrund unterschiedlicher Methoden jedoch nur zum Teil über die Zeit vergleichbar und eine generelle Einschätzung des Ausmaßes motorischer Auffälligkeiten ist dadurch erschwert [Landesinstitut für den Öffentlichen Gesundheitsdienst 1999].

Im Kinder- und Jugendgesundheitssurvey sollen über ausgewählte Indikatoren zentrale Dimensionen der Motorik einheitlich erfasst und eine Beschreibung der derzeitigen Situation ermöglicht werden. Die Darstellung von Zusammenhängen zwischen dem motorischen Status und anderen Untersuchungs- und Befragungsdaten kann zur Ableitung präventiver Maßnahmen führen. Die erhobenen Daten können die Basis für neue Referenzwerte bilden.

\section{Methode und Stichprobe}

Entsprechend der altersspezifischen Entwicklung wird in der Altersgruppe der 4- bis 10-jährigen Kinder die Koordination und Reaktion sowie die Beweglichkeit der Wirbelsäule überprüft, während bei den 11- bis 17-Jährigen die aerobe Ausdauerleistung getestet wird.

Das Untersuchungsspektrum für die jüngere Altersgruppe umfasst zwei Tests aus der Motorischen Leistungsserie nach Schoppe [Neuwirth 2001], die die Auge-Hand-Koordination und die Koordination unter Zeitdruck überprüfen (Linien nachfahren, Stifte umstecken), sowie einen Reaktionstest, bei dem die Reaktionsschnelligkeit auf einen optischen Reiz ermittelt wird. Der Einbeinstand auf einer T-Schiene dient der Überprüfung der Koordination bei Präzisionsaufgaben, und mit dem seitlichen Hinund Herspringen wird die Koordination unter Zeitdruck zusammen mit der dynamischen Kraftausdauer der Beinmuskulatur getestet. Die Beweglichkeit der Wirbelsäule wird durch die Rumpfbeuge beurteilt. Die Durchführung der Tests erfolgt standardisiert und mit einheitlichen Instruktionen.

Die aerobe Ausdauerleistung bei den älteren Kindern und Jugendlichen wird mittels Fahrradergometrie nach einem definierten Belastungsprotokoll beurteilt. Die Eingangsbelastung beträgt 0,5 Watt pro Kilogramm Körpergewicht und wird alle zwei Minuten bis zum Erreichen einer Pulsfrequenz von 180/min um weitere 0,5 Watt/kg gesteigert. Die Kontraindikationen zur Ergometrie [Washington et al. 1994] und die Abbruchkriterien während der Untersuchung [Hebestreit et al. 1997] sind genau festgelegt.

\section{Erfahrungen aus dem Pretest}

Im Pretest wurden weitere Einzeltests zur Beurteilung motorischer Grundfähigkeiten, die ebenfalls zu validen Ergebnissen geführt haben, erprobt [Kahl, Emmel 2002]. Durch ein dem Kinderund Jugendgesundheitssurvey angeschlossenes Motorik-Modul besteht die Möglichkeit, vertiefende Untersuchungen zur motorischen Leistungsfähigkeit durchzuführen. Dabei werden auch Untersuchungselemente, die sich im Pretest bewährt haben, eingesetzt [Bös et al. 2002].

\section{Untersuchung der Haut}

\section{Problemstellung}

Neben anderen akuten und chronischen Hauterkrankungen nimmt die atopische Dermatitis (atopisches Ekzem oder Neurodermitis) nicht nur wegen der erheblich belastenden Symptomatik, sondern auch aufgrund der steigenden Häufigkeit eine besondere Rolle ein. Über ihre Verbreitung im Kindes- und Jugendalter sind die Erkenntnisse unbefriedigend. Von [SchulzLarsen 1993] und im European White Paper [Aas et al. 1997] wird die Lebenszeitprävalenz in Europa mit 9 bis 24\%, im Durchschnitt $12 \%$ angegeben und eine erhebliche Zunahme in den zurückliegenden Jahrzehnten konstatiert. Im Allergie-Spezialbericht des Statistischen Bundesamtes [Wahn et al. 2000] finden sich für Deutschland in den letzten beiden Jahrzehnten Häufigkeiten zwischen 0,2 (Alter 5-18 Jahre) und 19,7\% (Alter 9-11 Jahre). Ein zeitlicher Trend ist nicht sicher auszumachen; insbesondere bieten die Daten keine Anhaltspunkte dafür, dass die 
schwereren Verlaufsformen der atopischen Dermatitis an Verbreitung zugenommen haben.

Der Kinder- und Jugendgesundheitssurvey wird erstmalig bundesweit repräsentative Daten zur Prävalenz der atopischen Dermatitis schaffen, mit dem Ziel, u. a. auch jahreszeitliche Schwankungen aufzudecken. Durch die konjunkte Erhebung mit anderen Daten können Hypothesen aufgestellt werden, die den Einfluss des atopischen Ekzems z. B. auf die Lebensqualität, die Inanspruchnahme medizinischer Leistungen und den Medikamentenkonsum beschreiben. Hinweise auf die Rolle der allergischen Sensibilisierung, der Ernährung im frühen Kindesalter, insbesondere des Stillens auf die atopische Dermatitis, werden erwartet.

\section{Methoden und Stichprobe}

Bei allen Kindern und Jugendlichen wird eine Inspektion der Haut vorgenommen. Symptome einer akuten atopischen Dermatitis werden standardisiert mit dem Scorad-Schema (scoring of atopic dermatitis) beurteilt. Dieses erfasst die Ausdehnung der Hauterkrankung und den Schweregrad der betroffenen Stellen als objektive Erkrankungsparameter sowie den Leidensdruck der Betroffenen als subjektive Erkrankungsparameter.

\section{Erfahrungen aus dem Pretest}

Neben der Neurodermitis wurden im Pretest auch die Möglichkeiten der Erfassung anderer Hautkrankheiten im Rahmen eines solchen Studiendesigns erprobt. Dabei erwies sich die atopische Dermatitis als das dermatologische Problem mit der größten Verbreitung (mehr als die Hälfte aller festgestellten Hauterkrankungen) und der sichersten Diagnostizierbarkeit. Darüber hinaus konnte die Erhebung standardisiert durchgeführt werden. Andere Hautprobleme und Fragestellungen bereiteten hingegen differenzialdiagnostische Probleme und erwiesen sich zudem als sehr zeitaufwändig.

\section{Anthropometrie}

\section{Problemstellung}

Durch die Verbesserung der allgemeinen Lebensbedingungen ist es zu einem säkularen Trend der körperlichen Entwicklung gekommen. Einzelstudien und Untersuchungen in vergleichbaren Ländern zeigen z. B. für die Körpergröße einen Anstieg um rund $20 \mathrm{~cm}$ in den zurückliegenden 100 Jahren, wobei sich der größte Teil der Größenzunahme bereits in den ersten Lebensjahren manifestiert [van Wieringen 1986]. In Deutschland fehlen bislang für das gesamte Kindes- und Jugendalter repräsentative anthropometrische Daten, die nach internationalen Standards erhoben wurden. Die Prävalenz von Übergewicht und Adipositas nimmt bei Kindern und Jugendlichen zu [Bergmann, Mensink 2000, Chinn, Rona 2001, Mokdad et al. 1999]. Der Bodymass-Index (BMI) bildet die übermäßige Vermehrung von Fettgewebe und dessen Verteilung nur unzureichend ab, daher sind zur Beurteilung des Körperhabitus neben Indikatoren für die fettfreie Körpermasse auch solche für das Fettgewebe und dessen Verteilung erforderlich. Als Folge von Essstörungen, die im Kindes- und Jugendalter eine zunehmende Rolle spielen [Holtkamp, HerpertzDahlmann 2002], können sowohl Übergewichtigkeit als auch Untergewichtigkeit vorkommen.
Durch den Kinder- und Jugendgesundheitssurvey werden für Deutschland repräsentative Daten für die Körpermaße erhoben, die Aussagen zur Verbreitung von Übergewicht und Adipositas sowie Untergewicht bei Kindern und Jugendlichen differenziert nach Alter, Geschlecht, Sozialstatus und anderen Variablen ermöglichen. Die Daten können darüber hinaus für regionale und internationale Vergleiche sowie für Trendbeobachtungen verwendet werden. Durch die gemeinsame Analyse mit anderen Befragungs-, Untersuchungs- und Labordaten können Zusammenhänge des Köperhabitus u. a. zum Geburtsgewicht, dem BMI der Eltern, der Ernährungsweise, den motorischen Fähigkeiten oder Risikofaktoren für Herz-Kreislauf-Krankheiten beschrieben werden.

\section{Methoden und Stichprobe}

Die Körpergröße wird, wenn das Kind noch nicht sicher stehen kann, im Liegen mithilfe eines Holtain-Infantometers, danach im Stehen (ohne Schuhe) mithilfe eines portablen Holtain-Stadiometers ermittelt. Das Körpergewicht wird bis zum Alter von zwei Jahren ohne Bekleidung auf einer digitalen Säuglingswaage, ab drei Jahren in leichter Bekleidung (Unterwäsche) ohne Schuhe auf einer elektronischen Stehwaage bestimmt. Die Hautfaltendicke über dem Triceps und subskapulär wird bei allen Kindern auf der rechten Seite mit einem Holtain-Caliper gemessen. Ab einem Alter von sechs Jahren wird zur Ermittlung des FRAME-Index die Epikondylenbreite des rechten Oberarms mit einem Tasterzirkel gemessen. Der Bauch- und Hüftumfang wird ab einem Alter von elf Jahren, der Kopfumfang in allen Altersstufen mit einem Textilmessband bestimmt.

\section{Reifestatus}

\section{Problemstellung}

Die Reifeentwicklung unterliegt, ähnlich wie die Körpergröße, einem säkularen Trend, der auch als Akzeleration bezeichnet wird. Der Reifestatus hat nicht nur eine herausragende Bedeutung für die Persönlichkeitsentwicklung und damit für das Verhalten, sondern ist auch für die Interpretation zahlreicher biologischer Messwerte (u. a. für Wachstum, Blutdruck, Blutlipide, Eisenstatus einschließlich rotem Blutbild) unentbehrlich.

\section{Methode und Stichprobe}

Für die Beurteilung der Reifeentwicklung werden bei allen Probanden ab 11 Jahren neben der Befragung zum Eintritt der Regelblutung bei Mädchen und des Stimmbruchs bei Jungen die international gebräuchlichen, definierten und in Bildtafeln dargestellten Entwicklungsstufen von Tanner [Tanner, Whitehouse 1976] verwendet. Aus diesem Spektrum wurde das Merkmal Schambehaarung ausgewählt, da es somatoskopisch einfach zu beurteilen ist.

\section{Erfahrungen aus dem Pretest}

Im Pretest wurde vor allem die Akzeptanz dieser den Intimbereich der Kinder und Jugendlichen betreffenden Untersuchung geprüft. Die Fragen zu Regelblutung und Stimmbruch wurden von den Kindern und Jugendlichen gut akzeptiert und führten zu aussagefähigen und validen Ergebnissen. Die Beurteilung der Entwicklungsstufen der Pubesbehaarung durch einen Arzt bzw. eine Ärztin wurde von fast der Hälfte der Probanden verweigert. 
Hingegen überforderte die Selbsteinschätzung der Schambehaarung, anhand der definierten Bildtafeln nach Tanner, das in der Altersgruppe der 11- bis 17-jährigen Mädchen und Jungen zum Teil sehr ausgeprägte Schamgefühl nicht und führte zu vollständigen und plausiblen Aussagen zum Reifestatus.

\section{Literatur}

${ }^{1}$ Aas K, Aberg N, Bachert C et al. European Allergy White Paper. Allergic diseases as a public health problem in Europe. The UCB Institute of Allergy, Bruxelles. Brussels: Presented to the European Parliament 28th June, 1997

2 Bergmann KE, Mensink GBM. Körpermaße und Übergewicht. BundesGesundheitssurvey 1998. Gesundheitswesen 2000; 61 (Sonderheft 2): $115-120$

${ }^{3}$ Bös K, Heel J, Romahn N et al. Untersuchungen zur Motorik im Rahmen des Kinder- und Jugendgesundheitssurveys. Gesundheitswesen 2002; 64 (Sonderheft 1): 80-87

${ }^{4}$ Chinn S, Rona RJ. Prevalence and trends in overweight and obesity in three cross sectional studies of British children, 1974-94. BMJ 2001; 322: $24-26$

${ }^{5}$ De Swiet M, Fayers P, Shinebourne EA. Blood pressure in the first 10 years of life: the Brompton study. BMJ 1992; 304 (6818): 23-26

${ }^{6}$ Etnier JL, Salazar W, Landers DM et al. The influence of physical fitness and exercise upon cognitive functioning: a meta-analysis. Journal of Sport and Exercise Psychologie 1997; 19: 249-277

${ }^{7}$ Gaschler P. Motorik von Kindern und Jugendlichen heute - Eine Generation von „Weicheiern, Schlaffis und Desinteressierten“? (Teil 1). Haltung und Bewegung 1999; 3: 5-16

${ }^{8}$ Hebestreit H, Lawrenz W, Zelger O et al. Ergometrie im Kindes- und Jugendalter. Monatsschr Kinderheilkd 1997; 145: 1326-1336

${ }^{9}$ Hohmann A, Haase W. Effektives Seh-Screening kann die Amblyopierate senken. Ophthalmologe 1993; 90: 2-5

${ }^{10}$ Holtkamp K, Herpertz-Dahlmann B. Anorexia und Bulimia nervosa im Kindes- und Jugendalter. Monatsschr Kinderheilkd 2002; 150: 164-171

${ }^{11}$ Kahl H, Emmel J. Der Untersuchungsteil Motorik im Pretest des Kinder- und Jugendgesundheitssurveys. Gesundheitswesen 2002; 64 (Sonderheft 1): 114-118

12 Landesinstitut für den Öffentlichen Gesundheitsdienst (LÖGD). NRW Dokumentation der schulärztlichen Untersuchungen NordrheinWestfalen 1999
${ }^{13}$ Lauer RM, Clarke WR. Childhood risk factors for high adult pressure: the Muscatine Study. Pediatrics 1989; 84: 633-641

${ }^{14}$ Mensink G, Deketh M, Mul MDM et al. Physical activity and its association with cardiovascular risk factors and mortality. Epidemiology 1996; 7: 391-397

${ }^{15}$ Mokdad AH, Serdula MK, Dietz WH et al. The spread of the obesity epidemic in the United States, 1991-1998. JAMA 1999; 282: 1519-1522

${ }^{16}$ Moore BD. Eye Care for Infants and Young Children. Boston: Butterworth 1998

${ }^{17}$ Nelson MJ, Ragland DR, Syme SL. Longitudinal prediction of adult pressure from juvenile blood pressure levels. Am J Epidemiol 1992; 136: 633-645

${ }^{18}$ Neuwirth W. Motorische Leistungsserie nach Schoppe (MLS) - Handanweisung, Version 23.00. Österreich: Verlag Dr. G. Schufried GmbH 2001

${ }^{19}$ Schulz-Larsen F. The epidemiology of atopic dermatitis. In: Burr ML (Hrsg). Epidemiology of Clinical Allergy. Basel. Monogr. Allergy: Karger 1993; 31: 9-28

${ }^{20}$ Sönnichsen C, Weineck J, Köstermeyer G. PEP - eine Studie zur Präventionserziehung - Teil 3: Motorisches Leistungsvermögen und kardiovaskuläres Risiko von Schulanfängern. Haltung und Bewegung 1997; 3: $5-10$

${ }^{21}$ Stallones L, Müller WH, Christensen BL. Blood pressure, fatness, and fat patterning among USA adolescents from two ethnic groups. Hypertension 1982; 4: 483-486

22 Tanner JM, Whitehouse R. Clinical longitudinal standards for height, weight, height velocity, weight velocity and the stages of puberty. Arch Dis Childh 1976; 51: 170

${ }^{23}$ Van Wieringen JC. Secular growth changes. In: Falkner F, Tanner JM (Hrsg). Human Growth. A Comprehensive Treatise. 2nd Edition. Vol III: Methodology, ecological, genetic, and nutritional effects on growth. New York: Plenum Press 1986; 307-331

${ }^{24}$ Wahn U, Wichmann HE, Bergmann R et al. Spezialbericht Allergien. Gesundheitsberichterstattung des Bundes. Stuttgart: Metzler und Pöschel Verlag 2000

${ }^{25}$ Washington RL, Bricker JT, Alpert BS et al. Guidelines for exercise testing in the pediatric age group. From the Committee on Atherosclerosis and Hypertension in Children, Council on Cardivascular Disease in the Young, the American Heart Association. Circulation 1994; 90: 2166-2179

${ }^{26}$ Zieske AW, Malcolm DT, Strong JP. Natural history and risk factors of arteriosclerosis in children and youth: the PDAY study. Pediatric Pathology and Molecular Medicine 2002; 21: 213-237 